\title{
Sputtering of silicon by low-energy oxygen bombardment studied by MD simulations
}

\author{
Patrick Philipp, ${ }^{a *}$ Tom Wirtz ${ }^{a}$ and John Kieffer ${ }^{b}$
}

\begin{abstract}
In the field of secondary ion mass spectrometry, ion-matter interactions have been largely investigated by numerical simulations. For molecular dynamics simulations related to inorganic samples, mostly classical force fields assuming stable bonding structure have been used. In this paper, we will use a reactive force field capable of simulating the breaking and formation of chemical bonds. Important features of this force field for simulating systems that undergo significant structural reorganisation are (i) the ability to account for the redistribution of electron density upon ionization, formation, or breaking of bonds, through a charge transfer term, and (ii) the fact that the angular constraints dynamically adjust when a change in the coordination number of an atom occurs. In this work, we present results obtained for the simulation of low-energy oxygen bombardment of crystalline and amorphous silicon. Information on variation of sputtering yields, energy, and angular distributions as well as the emission of clusters are studied. Compared to normal force fields, ion-matter interactions as well as the sputtering of matter should be described more accurately, especially when using reactive primary ions (oxygen or cesium) at low impact energies. Copyright $\odot 2012$ John Wiley \& Sons, Ltd.
\end{abstract}

Keywords: MD simulations; simulations; sputtering; cluster emission; reactive force field; silicon

\section{Introduction}

The characterisation of thin layered samples by low-energy secondary ion mass spectrometry (SIMS), e.g. for inorganic samples related to microelectronics applications or organic multilayered samples for optoelectronic devices, is only possible if the parameters influencing the analysis outcome are known and can be controlled. ${ }^{[1]}$ Most challenging issues are depth resolution for layers with $\mathrm{nm}$-scale thickness as well as ionization mechanisms depending on sample composition, concentration variations in between different layers, and varying surface concentration of the implanted primary ion species, either in the pre-equilibrium regime or in between two layers. ${ }^{[2,3]}$ Depth resolution as well as the matrix effect have been largely investigated and improved by experimental techniques.

Molecular dynamics (MD) simulations can contribute successfully by exploring the nanoscale mechanisms of sputtering and damage formation in the sample during ion bombardment. ${ }^{[4-6]}$ They have been successfully applied to describe sputtering and cascade formation for many applications, including monatomic and cluster bombardment of inorganic and organic samples. Cluster sizes range from small clusters containing few atoms to massive clusters of up to several thousand atoms. ${ }^{[4]}$ Applications vary from cascade formation during monatomic bombardment and the sputtering of organic fragments and molecules during SIMS analyses to surface smoothening and etching by massive clusters. Monatomic bombardment of Si surfaces is of particular interest for this work. Previous simulation studies report on sputter yields, ${ }^{[7,8]}$ angular and energy distributions, ${ }^{[7-9]}$ as well as damage formation. ${ }^{[10-13]}$ Most of the studies on ion bombardment are making use of classical force fields which assume stable bonding and either covalent or Coulomb interactions. A combination of both is not possible. Such simple potentials are not well suited for systems undergoing significant structural changes or including reactive species like oxygen or cesium. This is in particular true for sputtering by reactive species in SIMS. Reactive force fields have been developed to model such systems. In this paper, we will use the force field of Kieffer which has been modified for the simulation of sample irradiation by energetic ions. ${ }^{[14]}$ Previously, it has already been applied successfully to the simulation of structural and dynamic properties of cristiobalite ${ }^{[15,16]}$ and other systems. ${ }^{[17-19]}$

In this paper, we will give a short description of this force field and describe the implantation of the primary ion species as well as the sputtering of atoms and small clusters for $250 \mathrm{eV}$ and $500 \mathrm{eV}$ bombardment of a crystalline $\mathrm{Si}(100)$ and an amorphous Si surface by single oxygen atoms.

\section{Model}

Important features of the Kieffer force field are the ability to account for the formation and breaking of bonds. These mechanisms are modelled by a charge transfer term and the dynamic adjustment of the angular constraints when a change in the coordination number of a given atom occurs. It is a general potential that should be able to reproduce the properties of many systems and not only the specific properties of a welldefined system. The reactive three-body potential includes a Coulomb term, a Born-Huggins-Mayer repulsive term, as well as a three-body term:

\footnotetext{
* Correspondence to: Patrick Philipp, Science and Analysis of Materials, Centre de Recherche Public - Gabriel Lippmann. E-mail: philipp@lippmann.lu

a Department 'Science and Analysis of Materials' (SAM), Centre de Recherche Public - Gabriel Lippmann, 41 rue du Brill, L-4422 Belvaux, Luxembourg

b Department of Materials Science and Engineering, University of Michigan, Ann Arbor, Michigan, 48109-2136, USA
} 


$$
\begin{aligned}
\phi_{i}= & q_{i} \sum_{j=1}^{N} \frac{q_{j}}{4 \pi \varepsilon_{0} r_{i j}}+\sum_{j=1}^{N C} C_{i j} e^{\left(\sigma_{i}+\sigma_{j}-r_{i j}\right) \cdot \rho_{i j}} \\
& +\sum_{j=1}^{N C-1} \sum_{k=j+1}^{N C}\left(\varphi_{i j}+\varphi_{i k}\right)\left(4 e^{-\gamma_{i j k}\left(\bar{\theta}-\theta_{i j k}\right)^{4}}-3\right)
\end{aligned}
$$

where $\phi_{i}$ is the potential energy of the particle, $q_{i}$ is the charge, $\varepsilon_{0}$ is the dielectric constant of the vacuum, and $r_{i j}$ is the interatomic distance. Coulomb interactions are calculated by summing over $N$ atoms up to the cut-off distance of the Ewald summation, while the other interactions sum over NC neighbours up to the cut-off distance of the Born-Huggins-Mayer term. The charge of each atom is given by equation $q_{i}=q_{i}^{O}-\sum_{j=1}^{N C} \delta_{i j} \zeta_{i j}$, where $q_{i}^{0}$ is the charge of the isolated atom and $\zeta_{i j}=\frac{1}{1+e^{b\left(r_{i j}-a\right)}}$ is the charge transfer function. Both, $a$ and $b$ are empirical parameters. Covalent bonding is modelled by $\left(\varphi_{i j}+\varphi_{i k}\right)\left(4 e^{-\gamma_{i j k}\left(\bar{\theta}-\theta_{i j k}\right) 4}-3\right)$, where $\varphi_{i j}=-C_{i j} \frac{\kappa_{i j}}{\eta_{i j}} \zeta_{i j} e^{\left(\lambda_{i j}-r_{i j}\right) \eta_{i j}}$ models the attraction between two atoms, $\bar{\theta}$ is the equilibrium bond angle, and $\theta_{i j k}$ is the angle formed by the bond vectors $r_{i j}$ and $r_{i k}$. Furthermore, $C_{i j}=A_{i j}\left(1+\frac{z_{i}}{n_{i}}+\frac{z_{j}}{n_{j}}\right)$ where $z_{i}$ is the valence and $n_{i}$ is the maximum number of electrons in the outer shell of atom i. Coulomb interactions are calculated using the Ewald summation. For the low impact energies studied in this paper, an interpolation to a high-energy potential like the ZBL potential is not required. A more detailed description of the original force field can be found in. ${ }^{[14]}$ Modifications to that potential as well as the optimisation of $\mathrm{Si}-\mathrm{O}$ parameters are described in reference. ${ }^{[20]}$

MD simulations of amorphous $\mathrm{Si}$ and $\mathrm{Si}(100)$ surfaces have been carried out for impact energies of $250 \mathrm{eV}$ and $500 \mathrm{eV}$ and an incidence angle equal to $60^{\circ}$. This angle corresponds roughly to the incidence angle on the Cameca SC-Ultra instrument under the same experimental conditions. The azimuthal angle is chosen randomly. The amorphous sample has a size of $4.3 \cdot 4.3 \cdot 12.2 \mathrm{~nm}^{3}$ containing 10000 atoms while the crystalline sample is a 8.8.15 supercell containing 7680 atoms. The amorphous sample has been obtained by defining random atom positions at $2000 \mathrm{~K}$, relaxing the system for $8.5 \mathrm{~ns}$ at that temperature and ramping it during $4 \mathrm{~ns}$ down to $300 \mathrm{~K}$. At $300 \mathrm{~K}$, the system could relax during $1.5 \mathrm{~ns}$. Both samples form 2D slaps with periodic boundary conditions in $x-y$ directions. The current implementation of the Ewald summation does not allow removing them.

In order to obtain reliable statistics for every condition, 400 random impacts have been simulated on the pristine amorphous surface and 90 impacts on the crystalline surface. The simulations have been carried out at $300 \mathrm{~K}$ at constant volume and without applying any energy control. The time step was chosen such that the maximum energy drift stayed below $0.5 \%$.

\section{Results and discussion}

The simulation of oxygen bombardment of silicon surfaces should allow for the study of sputtering mechanisms during initial stages of sputtering (static regime) in amorphous and crystalline samples. For the crystalline sample, the surface atoms are reorganised in dimers, and thus more strongly bonded than those of the amorphous sample. One consequence of this can be observed in Fig. 1 where the stopping power averaged over the first $2.5 \AA$ at the sample surface is significantly higher

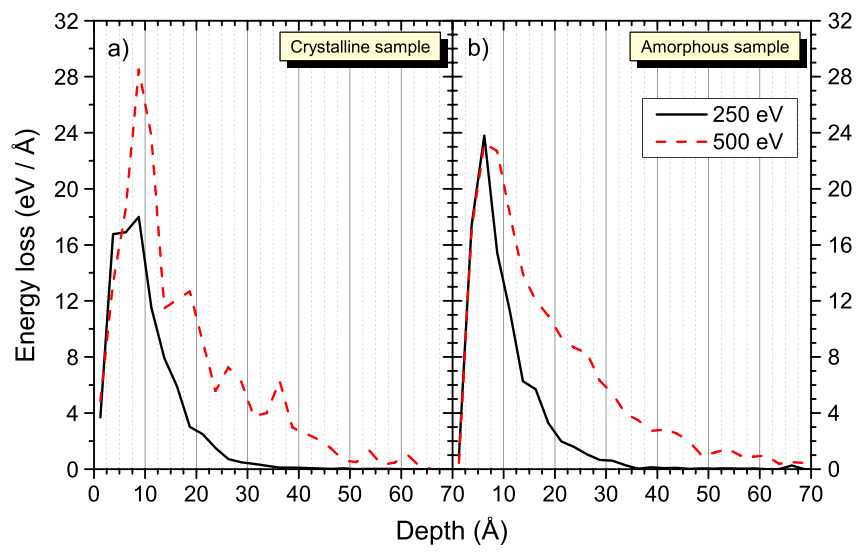

Figure 1. Stopping power with respect to depth for $250 \mathrm{eV}$ and $500 \mathrm{eV}$ oxygen bombardment of a) crystalline and b) amorphous Si samples.

(3.5 eV/ $\AA$ - $5.0 \mathrm{eV} / \AA$ ) than those of the amorphous sample $(0.5 \mathrm{eV} / \AA-1.0 \mathrm{eV} / \AA)$. For greater depths, it becomes more difficult to distinguish both samples. The distribution of the stopping power with depth is broader for the amorphous target, but the maximum is closer to the sample surface (in between $5.0 \AA$ and $7.5 \AA$ compared to in between $7.5 \AA$ and $10.0 \AA$ for the crystalline sample). At the same time, the distribution of the crystalline sample presents the longest tail. This behaviour can be explained by the smaller density of the amorphous target $\left(2.05 \mathrm{~g} / \mathrm{cm}^{3}\right.$ compared to $2.33 \mathrm{~g} / \mathrm{cm}^{3}$ for the crystalline target) which facilitates the implantation of the incoming oxygen atom and the low-density channels in the crystalline silicon. Although the direction of the bombarding atoms are not directly parallel to those channels, they may get deviated into them below the surface and continue then with reduced stopping power.

Those mechanisms also influence the depth of origin of the sputter silicon atoms (Fig. 2). For the crystalline sample at $250 \mathrm{eV}$ bombardment, $94 \%$ of the sputtered atoms originate from the first $2.5 \AA$ below the sample surface. This number drops to $76 \%$ for the amorphous silicon. At $500 \mathrm{eV}$, the situation is similar with $83 \%$ of the atoms of the crystalline sample being sputtered from the first $2.5 \AA$ compared to $65 \%$ for the amorphous target. For the crystalline target, all atoms are also sputtered from the two first atomic layers (about the first 5.0 $\AA$ ) while atoms of the amorphous sample can originate up to a depth of $7.5 \AA$ for

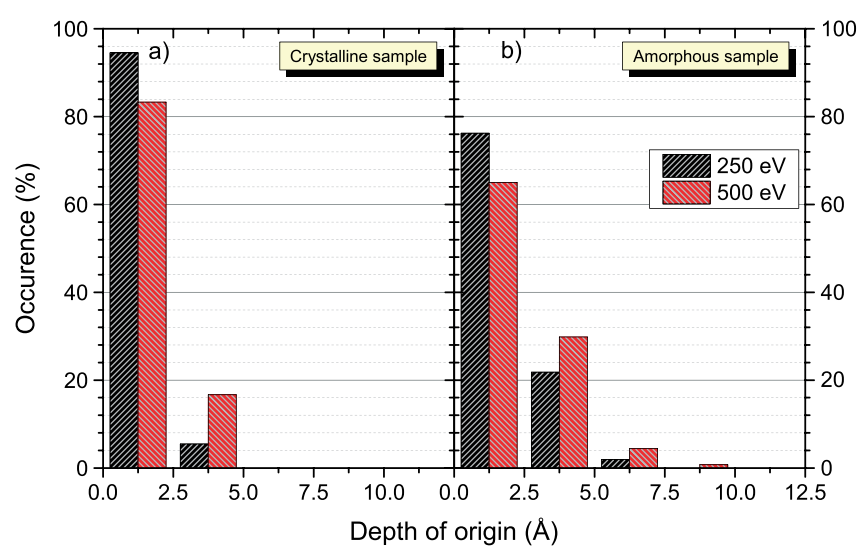

Figure 2. Depth of origin of sputtered matter for $250 \mathrm{eV}$ and $500 \mathrm{eV}$ oxygen bombardment of a) crystalline and b) amorphous Si samples. 
the $250 \mathrm{eV}$ and up to $10.0 \AA$ for the $500 \mathrm{eV}$ bombardment. Nevertheless, in both conditions, more than $90 \%$ of the atoms originate from the first $5.0 \AA$.

The sputtering yield depends both on the density and the crystallinity of the target. For the crystalline target, the reorganised $\mathrm{Si}(100)$ surface results in a significantly increased stopping power when compared to the amorphous sample. However, the sputtering yield is much lower (Fig. 3a). Silicon atoms of the amorphous sample are much more loosely bonded, and thus easier to sputter. Thus is reflected by the corresponding sputtering yields of 0.35 (crystalline sample) and 0.7 (amorphous sample) at $250 \mathrm{eV}$ as well as those of 0.5 (crystalline sample) and 1.0 (amorphous sample) at $500 \mathrm{eV}$. For both energies, the sputtering yields are lower than those predicted by Stopping and Range of lons in Matter (SRIM) $)^{[21,22]}(0.9$ at $250 \mathrm{eV}$ and 1.6 at $500 \mathrm{eV}$ ) The different sputtering yields correlate quite well with the different primary ion ranges (Fig. 3b). At $250 \mathrm{eV}$, the MD range of the amorphous target is slightly higher than the one of the crystalline target. The range predicted by SRIM is highest. At $500 \mathrm{eV}$, the lowest range is obtained by SRIM, while those of the MD simulations vary still with the inverse of the sputtering yield. The range of the MD simulations seem to increase faster than for SRIM. At the same time, the SRIM simulations have been carried out with the theoretical density of silicon $\left(2.33 \mathrm{~g} / \mathrm{cm}^{3}\right)$ while the density of the amorphous target is significantly lower $\left(2.05 \mathrm{~g} / \mathrm{cm}^{3}\right)$.

For the emission of clusters, sample density and structure are also of large importance. They have been detected by analyzing the neighbour list at the end of the simulation. ${ }^{[23]}$ For both samples, the largest amount of matter is sputtered as single atoms (Fig. 4). For the crystalline sample, this value changes from $74 \%$ to $82 \%$ and for the amorphous sample from $78 \%$ to $81 \%$. Thus, both kinds of targets behave there in the same way. This changes for the emission of clusters. For the crystalline sample, only dimers have been emitted. For the amorphous sample, also larger clusters are observed. For the $250 \mathrm{eV}$ bombardment, 18\% of the atoms are emitted as $\mathrm{Si}_{2}$ and $2 \%$ as $\mathrm{Si}_{3}$ clusters. For the $500 \mathrm{eV}$ bombardment, even larger clusters have been observed. $19 \%$ of the matter is sputter as $\mathrm{Si}_{2}, 3.8 \%$ as $\mathrm{Si}_{3}$, and $0.2 \%$ as $\mathrm{Si}_{4}$ clusters. The last number represents a single event. Thus, larger clusters are emitted more easily from the less dense amorphous

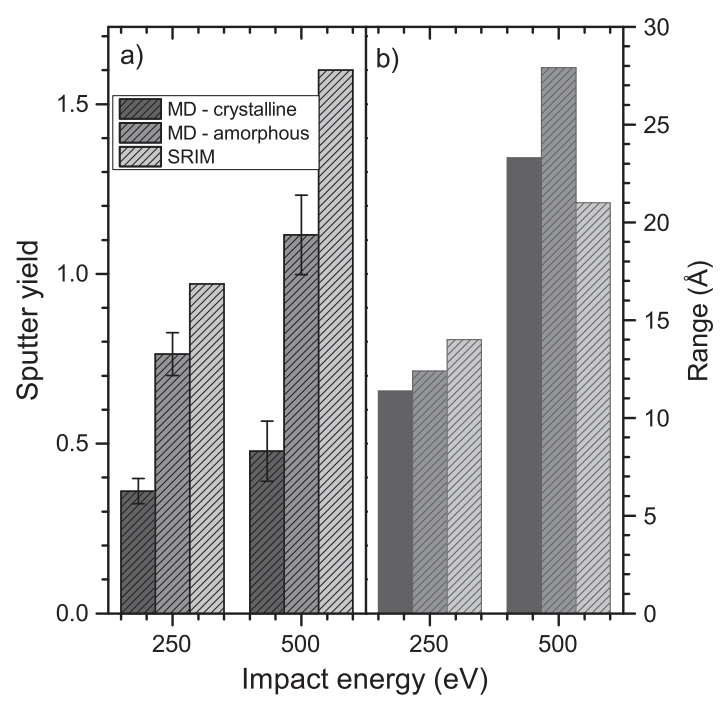

Figure 3. Comparison of a) different sputter yields and b) ranges for MD and SRIM simulations.

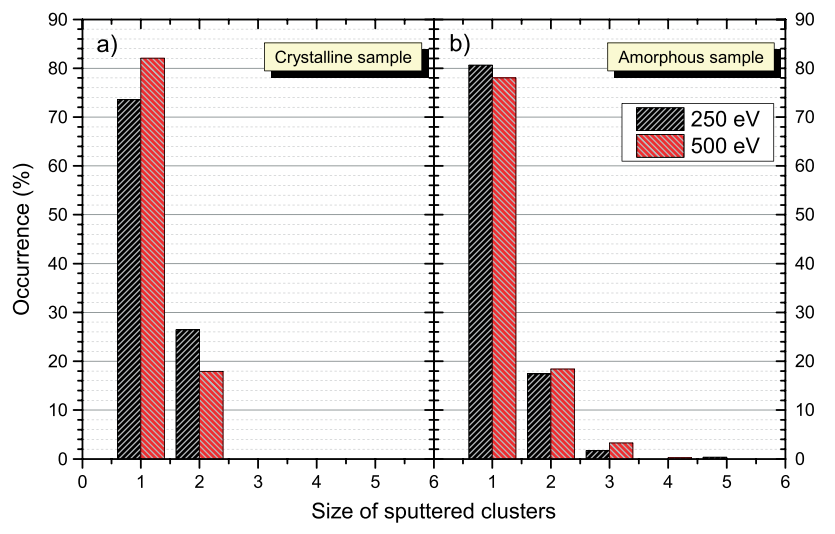

Figure 4. Size distribution of sputtered particles for $250 \mathrm{eV}$ and $500 \mathrm{eV}$ oxygen bombardment of a) crystalline and b) amorphous Si samples.

target. All clusters are also emitted via direct ejection. For Si clusters, direct ejection is expected, ${ }^{[7]}$ but might be increased by the too high bond energy in $\mathrm{Si}_{2}$ clusters $(4 \mathrm{eV}$ with our potential compared to the experimental value of $2.2 \mathrm{eV}) .{ }^{[7]}$ Yet, total cluster abundances qualitatively agree with data found in literature. For metals, simulations predict that sputtered clusters contribute in between $2 \%$ to several $10 \%$ to the total sputtered flux. ${ }^{[23-25]}$ Similar values are found in experiments. ${ }^{[26,27]}$ To our knowledge, for silicon, the only data available is on Xe and organic cluster bombardment. Xe bombardment results in cluster emission up to $20 \%$ while less clusters are ejected for the lighter organic primary clusters. ${ }^{[28]}$ However, in these experiments, secondary ions have been detected without multi-phonon ionization so that the results are affected by the cluster ionization probabilities.

The emission of $\mathrm{Si}_{n}-\mathrm{O}$ clusters has also been studied. As the simulation studies include only single oxygen atom bombardment of a pristine silicon surface, clusters containing more than one oxygen atom cannot be formed. Furthermore, clusters with more than one Si atom have not been observed, at least not for the 400 impacts carried out for every experimental condition. Thus, the probability to form such clusters must be rather small. For the crystalline sample, only one cluster has been observed for the $250 \mathrm{eV}$ bombardment. For the amorphous sample, more clusters have been observed, but the number of simulated impacts is also larger (400 impacts compared to 90 for the crystalline target). Five clusters have been observed for $250 \mathrm{eV}$ bombardment and two for the $500 \mathrm{eV}$ bombardment. Thus, the emission of $\mathrm{Si}$ - O clusters seems to be equally probably for amorphous and crystalline samples, at least for this number of simulated impacts. In every situation, the cluster is also formed between the backscattered oxygen atom and a silicon atom from the top atomic layer. The oxygen atom lifts this atom up while crossing the upper atomic layer. Before, the oxygen atom can either have been implanted down to few $\AA$, or has been forming a cascade close to the sample surface.

\section{Conclusions}

By studying the bombardment of both an amorphous and a crystalline silicon surface by $250 \mathrm{eV}$ and $500 \mathrm{eV}$ oxygen bombardment, the initial stages of sputtering could be modelled. Not surprisingly, the sputtering yield revealed to depend on the sample crystallinity and density. In a well-organised structure 
close to the optimal configuration, the atoms are more strongly bonded and thus more difficult to displace. Accordingly, the stopping power of the reorganised $\mathrm{Si}(100)$ surface is higher than the one of the amorphous sample and the irradiation of this surface results in smaller implantation depths and lower sputtering yields. Furthermore, for the amorphous target, sputtered atoms can originate from larger depths and the amount of sputtered $\mathrm{Si}-\mathrm{O}$ clusters is increased. No significant difference could be observed for $\mathrm{Si}_{2}$ clusters while $\mathrm{Si}_{n}$, with $\mathrm{n}>2$ are more abundant for the amorphous target. Nevertheless, in our simulations, the silicon atoms in sputtered $\mathrm{Si}$ - O clusters originate always from the first atomic layer.

\section{Acknowledgements}

This work has been supported by the National Research Fund, Luxembourg (NSF-FNR-MAT-07-01).

\section{References}

[1] K. Q. Ngo, P. Philipp, Y. Jin, S. E. Morris, M. Shtein, J. Kieffer, T. Wirtz, Surf. Interface Anal. 2011, 43, 194.

[2] H. A. Storms, K. F. Brown, J. D. Stein, Anal. Chem. 1977, 49(13), 2023.

[3] K. Wittmaack, Surf. Sci. 1983, 126, 573.

[4] B. J. Garrison, Z. Postawa, Mass Spectrom. Rev. 2008, 27, 289.

[5] H. Hansen, A. Rdinger, S. Messlinger, G. Stoian, Y. Rosandi, H. M. Urbassek, U. Linke, T. Michely, Phys. Rev. B 2006, 73, 235414.
[6] J. Samela, K. Nordlund, Nucl. Instrum. Methods Phys. Res. B 2007, 263, 375

[7] R. A. Stansfield, K. Broomfield, D. C. Clary, Phys. Rev. B 1989, 39, 7680.

[8] R. Smith, D. E. Harrison, B. J. Garrison, Phys. Rev. B 1989, 40(1), 93.

[9] D. E. Sanders, K. B. S. Prasad, J. S. Burnham, B. J. Garrison, Phys. Rev. B 1994, 50, 5358.

[10] R. Pinzon, H. M. Urbassek, Phys. Rev. B 2001, 63, 195319.

[11] M. Koster, H. M. Urbassek, Surf. Sci. 2002, 496, 196.

[12] K. Kyuno, D. G. Cahill, R. S. Averback, J. Tarus, K. Nordlund, Phys. Rev. Lett. 1999, 83, 4788.

[13] J. Nord, K. Nordlund, J. Keinonen, Phys. Rev. B 2002, 65, 165329.

[14] L. P. Huang, J. Kieffer, J. Chem. Phys. 2003, 118, 1487.

[15] L. P. Huang, J. Kieffer, Phys. Rev. B 2004, 69, 224203.

[16] L. P. Huang, J. Kieffer, Phys. Rev. B 2004, 69, 224204.

[17] L. P. Huang, J. Kieffer, Phys. Rev. B 2006, 74, 224107.

[18] L. P. Huang, M. Durandurdu, J. Kieffer, J. Phys. Chem. C 2007, 111, 13712.

[19] J. H. Zhou, J. Kieffer, J. Phys. Chem. C 2008, 112, 3473.

[20] P. Philipp, L. Briquet, T. Wirtz, J. Kieffer, Nucl. Instrum. Methods Phys. Res. B 2011, 269, 1555.

[21] J. P. Biersack, L. G. Haggmark, Nucl. Instrum. Methods 1980, 174, 257.

[22] J. P. Biersack, W. Eckstein, Appl. Phys. A-Matter 1984, 34, 73.

[23] G. Betz, W. Husinsky, Phil. Trans. R. Soc. Lond. A 2004, 362, 177.

[24] G. Betz, W. Husinsky, Nucl. Instrum. Methods Phys. Res. B 1995, 102, 281.

[25] T. Colla, H. M. Urbassek, A. Wucher, C. Staudt, R. Heinrich, B. J. Garrison, C. Dandachi, G. Betz, Nucl. Instrum. Methods Phys. Res. B 1998, 143, 284.

[26] C. Staudt, R. Heinrich, A. Wucher, Nucl. Instrum. Methods Phys. Res. B 2000, 164-165, 677.

[27] A. Wucher, M. Wahl, Nucl. Instrum. Methods Phys. Res. B 1996; 115, 581.

[28] H. Yamamoto, H. Asaoka, Appl. Surf. Sci. 2001, 169-170, 305. 